\title{
Comparative Study of Various Iterative Numerical Methods for Computation of Approximate Root of the Polynomials
}

\author{
Roopa K M, Venkatesha P
}

\begin{abstract}
The aim of this article is to present a brief review and a numerical comparison of iterative methods applied to solve the polynomial equations with real coefficients. In this paper, four numerical methods are compared, namely: Horner's method, Synthetic division with Chebyshev method (Proposed Method), Synthetic division with Modified Newton Raphson method and Birge-Vieta method which will helpful to the readers to understand the importance and usefulness of these methods.

Keywords: Horner's method, Synthetic division, Chebyshev method, Modified Newton Raphson method and Birge-Vieta method.
\end{abstract}

\section{INTRODUCTION}

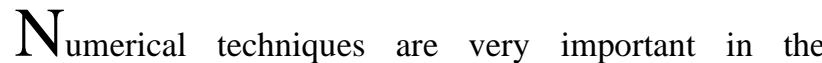
applications of Scientific and Engineering fields. It is also broadly used in all the major scientific disciplines. This article provides a brief on the comparative study of different methods for Numerical method to find the positive and negative roots of the polynomial equations by using various iterative techniques such as Horner's method, Synthetic division, Chebyshev method, Modified Newton-Raphson method and Birge-Vieta method

\section{METHODOLOGY}

\section{Synthetic Division method:}

Synthetic division is one of the method used to manually perform division of polynomials. The division of polynomials can also be done using the long division method. But, compared to the method of long division of polynomials, synthetic division requires less writing and fewer shortest method compared to the traditional long division of a polynomial for the special cases were the division by a linear factor.

Step-1: Consider the given polynomial say $P(x)$

Step-2: Arrange the coefficient of give polynomial equation in decreasing order

Manuscript received on November 23, 2021.

Revised Manuscript received on November 29, 2021.

Manuscript published on December 30, 2021.

* Correspondence Author

Dr. Roopa K M*, Department of Mathematics, Bangalore Institute of Technology, Bengaluru, India. Email: roopakm@bit-bangalore.edu.in

Venkatesha P, Department of Mathematics, Sri Sairam College of Engineering, Bengaluru, India. Email: venky.preethu743@ gmail.com

(C) The Authors. Published by Blue Eyes Intelligence Engineering and Sciences Publication (BEIESP). This is an open access article under the CC BY-NC-ND license (http://creativecommons.org/licenses/by-nc-nd/4.0/) calculations. This means that the synthetic division is the

Step-3: Perform the synthetic division with initial value $p_{0}$ Suppose, $P(x)=a_{0} x^{n}+a_{1} x^{n-1}+a_{2} x^{n-2}+\cdots \ldots+a_{n}$. Then synthetic division process is as follows:

\begin{tabular}{l|cccccc}
$p_{0}$ & $a_{0}$ & $a_{1}$ & $a_{2}$ & $\ldots \ldots \ldots \ldots$ & $a_{n-1}$ & $a_{n}$ \\
& & $p_{0} a_{0}$ & $p_{0} b_{1}$ & $\ldots \ldots \ldots \ldots$ & $p_{0} b_{n-2}$ & $p_{0} b_{n-1}$ \\
\hline & $b_{0}$ & $b_{1}$ & $b_{2}$ & $\ldots \ldots \ldots \ldots \ldots$ & $b_{n-1}$ & $b_{n}$ \\
& & $p_{0} b_{0}$ & $p_{0} c_{1}$ & $\ldots \ldots \ldots \ldots$ & $p_{0} c_{n-2}$ & \\
\hline & $c_{0}$ & $c_{1}$ & $c_{2}$ & $\ldots \ldots \ldots \ldots$ & $c_{n-1}$ &
\end{tabular}

\section{Horner's method:}

This is one of the traditional method to finding approximate values of both rational and irrational roots of the polynomial equations. Horner's method consists in diminution of the root of any equation by successive digits occurring in the roots. First, we have to find the integer part and then find decimal part to any desired decimal place of accuracy.

The step-by-step procedure for diminution is given below:

(i) Let $f(x)$ denotes the given polynomial.

Find $(a, b)$ for which $f(x)$ at $x=a$ and $x=b$ are the opposite signs.

(ii)Using $a$ we have to diminish the root of the equation $f(x)=0$ to get new equation $f_{1}(x)=0$ with root $0 \cdot d_{1} d_{2} d_{3} d_{4} \ldots \ldots \ldots$

(iii) The root of the equation $f_{1}(x)=0$ is multiply by 10 , so that now equation $g(x)=0$ is obtained with one of the root as $d_{1}{ }^{*} d_{2} d_{3} d_{4} \ldots \ldots \ldots$ which lies between 0 and 10 . Thus first figure after the decimal place is $d_{1}$.

(iv) Now, $d_{1}$ is found such that $g(x)$ at $x=d_{1}$ and $x=\left(d_{1}+1\right)$ are of the opposite signs. Then, the root of the equation $g(x)=0$ is diminished by $d_{1}$ and the resulting equation $g_{1}(x)=0$ with root $0 \times d_{2} d_{3} d_{4} \ldots \ldots \ldots$

(v) The root of the equation $g_{1}(x)=0$ is multiply by 10 , so that now equation $h(x)=0$ is obtained with one of the root as $d_{2}{ }^{*} d_{3} d_{4} \ldots \ldots \ldots$ which lies between 0 and 10 . Thus the second figure after the decimal place is $d_{2}$.

Continue this procedure to gain the approximate root of the equation to any desired degree of accuracy digit by digit.

3. Synthetic Division with Chebyshev method (Proposed Method):

This Proposed method is used to determine a real root or extract linear factor of the polynomial equation with minimum iterations while compared to other three methods. Algorithm for Synthetic Division with Chebyshev Method: Follow Step-1 to Step-3 of synthetic division method

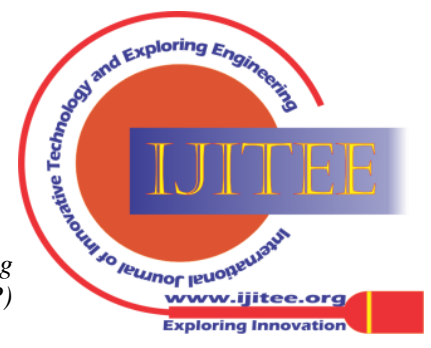




\section{Comparative Study of Various Iterative Numerical Methods for Computation of Approximate Root of the Polynomials}

Step-4: Find the next approximate root by

$\mathrm{x}_{\mathrm{k}+1}=\mathrm{x}_{\mathrm{k}}-\left\{\frac{\mathrm{f}\left(\mathrm{x}_{\mathrm{k}}\right)}{\mathrm{f}^{\prime}\left(\mathrm{x}_{\mathrm{k}}\right)}\right\}-\frac{1}{2}\left[\frac{\left\{\mathrm{f}\left(\mathrm{x}_{\mathrm{k}}\right)\right\}^{2} \mathrm{f}^{\prime \prime}\left(\mathrm{x}_{\mathrm{k}}\right)}{\left\{\mathrm{f}^{\prime}\left(\mathrm{x}_{\mathrm{k}}\right)\right\}^{3}}\right]$ for $k=0,1,2,3$

\begin{tabular}{|l|c|c|c|c|}
\hline & - & 8 & 368 & \\
\hline \multirow{2}{*}{8} & 1 & 46 & 3372 & \\
\cline { 2 - 3 } & - & 8 & & \\
\hline & 1 & 54 & & \\
\hline
\end{tabular}

Step-5: Repeat the Step-3 and Step-4 until $x$ reach desired accuracy.

\section{Synthetic division with Modified Newton Raphson method:}

It produces successively better approximations to the roots of a function.

Algorithm for Synthetic Division with Modified Newton

Raphson Method:

Follow Step-1 to Step-3 of synthetic division method

Step-4: Find the next approximate root by

$\mathrm{x}_{\mathrm{k}+1}=\mathrm{x}_{\mathrm{k}}-\left\{\frac{\mathrm{f}\left(\mathrm{x}_{\mathrm{k}}\right) \mathrm{f}^{\prime}\left(\mathrm{x}_{\mathrm{k}}\right)}{\left\{\mathrm{f}^{\prime}\left(\mathrm{x}_{\mathrm{k}}\right)\right\}^{2}-\mathrm{f}\left(\mathrm{x}_{\mathrm{k}}\right) \mathrm{f}^{\prime \prime}\left(\mathrm{x}_{\mathrm{k}}\right)}\right\}$ for $k=0,1,2,3 \ldots$

Thus, the transformed equation

$f_{2}(x)=x^{3}+54 x^{2}+3372 x-968=0$ has a root $0 \cdot \mathrm{d}_{2} \mathrm{~d}_{3} \mathrm{~d}_{4} \ldots \ldots \ldots \ldots$ which occurs in the interval $(0,1)$. Now, multiply the root of $f_{2}(x)=0$ by 10 then, the resultant equation $h(x)=x^{3}+540 x^{2}+337200 x-968000=0$ has one of its roots as $d_{2}{ }^{*} d_{3} d_{4} \ldots \ldots \ldots \ldots$ We can see that $\mathrm{h}(2)<0$ and $\mathrm{h}(3)>0$ so that $\mathrm{d}_{2}=2$.

III iteration: Now, using 2 we have to diminish the root of the equation $\mathrm{h}(\mathrm{x})=0$

\begin{tabular}{|c|c|c|c|c|}
\hline 2 & 1 & 540 & 337200 & -968000 \\
\cline { 2 - 4 } & - & 2 & 1084 & 676568 \\
\hline 2 & 1 & 542 & 338284 & -291432 \\
\cline { 2 - 4 } & - & 2 & 1088 & \\
\hline 2 & 1 & 544 & 339372 & \\
\cline { 2 - 3 } & - & 2 & & \\
\hline & 1 & 546 & & \\
\hline
\end{tabular}

Thus, the transformed equation $f_{3}(x)=x^{3}+546 x^{2}+339372 x-291432=0$ has a root $0 \cdot d_{3} d_{4} \ldots \ldots \ldots$ which occurs in the interval $(0,1)$. Now, multiply the root of $f_{3}(x)=0$ by 10 then, the resultant equation

$\mathrm{p}(\mathrm{x})=\mathrm{x}^{3}+5460 \mathrm{x}^{2}+33937200 \mathrm{x}-291432000=0$ has one of its roots as $d_{2}{ }^{\circ} d_{4} \ldots \ldots \ldots \ldots$ We can see that $p(8)<0$ and $p(9)>0$ so that this root is $8 d_{4} \ldots \ldots$

\section{CASE STUDY}

Case Study 1: Compute the root of the equation $\mathrm{x}^{3}+24 \mathrm{x}-50=0$.

IV iteration: Now, using 8 we have to diminish the root of the equation $\mathrm{p}(\mathrm{x})=0$

\section{Solution:}

Horner's method: Here $f(x)=x^{3}+24 x-50=0$ then, $\mathrm{f}(1)=-25<0$ and $\mathrm{f}(2)=6>0$

$\Rightarrow f(x)=0$ having one of the root in the interval $(1,2)$.

So, the integral (integer) part of the root of $f(x)=0$ is 1 .

I iteration: Now, using 1 we have to diminish the root of the equation $\mathrm{f}(\mathrm{x})=0$, so that the root of the transformed equation $0 \times \mathrm{d}_{1} \mathrm{~d}_{2} \mathrm{~d}_{3} \mathrm{~d}_{4 \ldots \ldots \ldots} \ldots$

\begin{tabular}{|c|c|c|c|c|}
\hline \multirow{2}{*}{1} & 1 & 0 & 24 & -50 \\
\cline { 2 - 4 } & - & 1 & 1 & 25 \\
\hline \multirow{2}{*}{1} & 1 & 1 & 25 & -25 \\
\cline { 2 - 4 } & - & 1 & 2 & \\
\cline { 2 - 4 } & 1 & 2 & 27 & \\
\hline & 1 & 1 & & \\
\hline
\end{tabular}

Thus, the transformed equation $f_{1}(x)=x^{3}+3 x^{2}+27 x-25=0$ has a root $0 \cdot d_{1} d_{2} d_{3} d_{4} \ldots \ldots \ldots$. which occurs in the interval $(0,1)$. Now, multiply the root of $f_{1}(x)=0$ by 10 so that the changed equation $\mathrm{g}(\mathrm{x})=\mathrm{x}^{3}+30 \mathrm{x}^{2}+2700 \mathrm{x}-25000=0$ has one of its roots as $d_{1}{ }^{\circ} d_{2} d_{3} d_{4} \ldots \ldots \ldots \ldots$ We see that $\mathrm{g}(8)<0$ and $\mathrm{g}(9)>0$ so that $\mathrm{d}_{1}=8$

II iteration: Now, using 8 we have to diminish the root of the equation $\mathrm{g}(\mathrm{x})=0$

\begin{tabular}{|c|c|c|c|c|}
\hline \multirow{2}{*}{8} & 1 & 30 & 2700 & -25000 \\
\cline { 2 - 5 } & - & 8 & 304 & 24032 \\
\hline 8 & 1 & 38 & 3004 & -968 \\
\hline
\end{tabular}

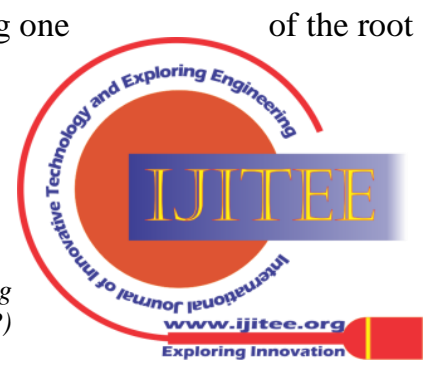




\section{I iteration:}

From the first iteration of Horner's method,

$\mathrm{f}(1)=-25, \mathrm{f}^{\prime}(1)=27, \frac{1}{2 !} \mathrm{f}^{\prime \prime}(1)=3 \Rightarrow \mathrm{f}^{\prime \prime}(1)=6$

Chebyshev method: $\mathrm{x}_{1}=\mathrm{x}_{0}-\left\{\frac{\mathrm{f}\left(\mathrm{x}_{0}\right)}{\mathrm{f}^{\prime}\left(\mathrm{x}_{\mathrm{n}}\right)}\right\}-\frac{1}{2}\left[\frac{\left[\mathrm{f}\left(\mathrm{x}_{0}\right)\right\}^{2} \mathrm{f}^{\prime \prime}\left(\mathrm{x}_{0}\right)}{\left\{\mathrm{f}^{\prime}\left(\mathrm{x}_{\mathrm{n}}\right)\right]^{3}}\right]$

$=1-\left\{\frac{-25}{27}\right\}-\frac{1}{2}\left[\frac{[-25\}^{2}(6)}{\{27\}^{3}}\right]=1+0.926-0.095=1.831$

II iteration:

\begin{tabular}{|c|c|c|c|c|}
\hline \multirow{2}{*}{1.831} & 1 & 0 & 24 & -50 \\
\cline { 2 - 4 } & - & 1.831 & 3.353 & 50.083 \\
\hline \multirow{2}{*}{1.831} & 1 & 1.831 & 27.353 & 0.083 \\
\cline { 2 - 4 } & - & 1.831 & 6.705 & \\
\hline \multirow{2}{*}{1.831} & 1 & 3.662 & 34.058 & \multirow{2}{*}{} \\
\cline { 2 - 3 } & - & 1.831 & & \\
\hline & 1 & 5.493 & & \\
\hline
\end{tabular}

$\mathrm{f}(1.831)=0.083, \mathrm{f}^{\prime}(1.831)=34.058, \frac{1}{2 !} \mathrm{f}^{\prime \prime}(1.831)=$ 5.493

$\Rightarrow \mathrm{f}^{\prime \prime}(1.831)=10.986$

Chebyshev method: $x_{2}=x_{1}-\left\{\frac{f\left(x_{1}\right)}{f^{\prime}\left(x_{1}\right)}\right\}-\frac{1}{2}\left[\frac{\left\{f\left(x_{1}\right)\right\}^{2} f^{\prime \prime}\left(x_{1}\right)}{\left\{f^{\prime}\left(x_{1}\right)\right\}^{3}}\right]$

$=1.831-\left\{\frac{0.083}{34.058}\right\}-\frac{1}{2}\left[\frac{[0.083\}^{2}(10.986)}{\{34.058\}^{3}}\right]$

$=1.831-0.002-0.000$

$\Rightarrow \mathrm{x}_{2}=1.829$

III iteration:

\begin{tabular}{|c|c|c|c|c|}
\hline \multirow{2}{*}{1.829} & 1 & 0 & 24 & -50 \\
\cline { 2 - 4 } & - & 1.829 & 3.345 & 50.014 \\
\hline \multirow{2}{*}{1.829} & 1 & 1.829 & 27.345 & 0.014 \\
\cline { 2 - 4 } & - & 1.829 & 6.690 & \\
\hline \multirow{2}{*}{1.829} & 1 & 3.658 & 34.035 & \\
\cline { 2 - 3 } & - & 1.829 & & \\
\hline & 1 & 5.487 & & \\
\hline
\end{tabular}

$\mathrm{f}(1.829)=0.014, \mathrm{f}^{\prime}(1.829)=34.035, \frac{1}{2 !} \mathrm{f}^{\prime \prime}(1.829)=$ $5.487 \Rightarrow \mathrm{f}^{\prime \prime}(1.831)=10.974$

Chebyshev method: $x_{3}=x_{2}-\left\{\frac{f\left(x_{2}\right)}{f^{\prime}\left(x_{2}\right)}\right\}-\frac{1}{2}\left[\frac{\left[f\left(x_{2}\right)\right]^{2} f^{\prime \prime}\left(x_{2}\right)}{\left\{f^{\prime}\left(x_{2}\right)\right\}^{3}}\right]$

$=1.829-\left\{\frac{0.014}{34.035}\right\}-\frac{1}{2}\left[\frac{[0.014\}^{2}(10.974)}{\{34.035\}^{3}}\right]$

$=1.829-0.000-0.000 \Rightarrow x_{3}=1.829$

\section{Synthetic division with Modified Newton Raphson} method:

Here $f(x)=x^{3}+24 x-50=0 \Rightarrow f^{\prime}(x)=3 x^{2}+24$ and $\mathrm{f}^{\prime \prime}(\mathrm{x})=6 \mathrm{x}$.

We know that $f(x)=0$ having one of the root in the interval $(1,2)$.

I iteration: From the I iteration of Horner's method, $\mathrm{f}(1)=-25, \mathrm{f}^{\prime}(1)=27, \frac{1}{2 !} \mathrm{f}^{\prime \prime}(1)=3 \Rightarrow \mathrm{f}^{\prime \prime}(1)=6$ From Modified Newton Raphson method,

$x_{1}=x_{0}-\left\{\frac{f\left(x_{0}\right) f^{\prime}\left(x_{0}\right)}{\left\{f^{\prime}\left(x_{n}\right)\right\}^{2}-f\left(x_{n}\right) f^{\prime \prime}\left(x_{n}\right)}\right\}=1-\left\{\frac{(-25)(27)}{\{27]^{2}-(-25)(6)}\right\}$ $=1+0.768=1.768$

II iteration:

\begin{tabular}{|c|c|c|c|c|}
\hline \multirow{2}{*}{1.768} & 1 & 0 & 24 & -50 \\
\cline { 2 - 5 } & - & 1.768 & 3.126 & 47.959 \\
\hline
\end{tabular}

\begin{tabular}{|c|c|c|c|c|}
\hline \multirow[t]{2}{*}{1.768} & 1 & 1.768 & 27.126 & \multirow[t]{5}{*}{-2.041} \\
\hline & - & 1.768 & 6.252 & \\
\hline \multirow[t]{3}{*}{1.768} & 1 & 3.536 & \multirow[t]{3}{*}{33.378} & \\
\hline & - & 1.768 & & \\
\hline & 1 & 5.304 & & \\
\hline
\end{tabular}

From Modified Newton Raphson method,

$x_{2}=x_{1}-\left\{\frac{f\left(x_{1}\right) f^{\prime}\left(x_{1}\right)}{\left\{f^{\prime}\left(x_{1}\right)\right\}^{2}-f\left(x_{1}\right) f^{\prime \prime}\left(x_{1}\right)}\right\}=1-$

$\left\{\frac{(-2.041)(33.378)}{\{33.378\}^{2}-(-2.041)(10.908)}\right\}$

$=1.768+0.06=1.828$

\section{III iteration:}

\begin{tabular}{|c|c|c|c|c|}
\hline \multirow{2}{*}{1.828} & 1 & 0 & 24 & -50 \\
\cline { 2 - 4 } & - & 1.828 & 3.342 & 49.981 \\
\hline \multirow{2}{*}{1.828} & 1 & 1.828 & 27.342 & -0.019 \\
\cline { 2 - 4 } & - & 1.828 & 6.684 & \\
\hline & 1 & 3.656 & 34.026 & \\
\cline { 2 - 3 } & - & 1.828 & & \\
\hline & 1 & 5.484 & & \\
\hline
\end{tabular}

$\mathrm{f}(1.828)=-0.019, \mathrm{f}^{\prime}(1.828)=34.026, \frac{1}{2 !} \mathrm{f}^{\prime \prime}(1.828)=$ $5.484 \Rightarrow f^{\prime \prime}(1.828)=10.968$

From Modified Newton Raphson method,

$\mathrm{x}_{3}=\mathrm{x}_{2}-\left\{\frac{\mathrm{f}\left(\mathrm{x}_{2}\right) \mathrm{f}^{\prime}\left(\mathrm{x}_{2}\right)}{\left\{\mathrm{f}^{\prime}\left(\mathrm{x}_{2}\right)\right\}^{2}-\mathrm{f}\left(\mathrm{x}_{2}\right) \mathrm{f}^{\prime \prime}\left(\mathrm{x}_{2}\right)}\right\}=1-$

$(-0.019)(34.026)$

$\left\{\overline{\{34.026\}^{2}-(-0.019)(10.968)}\right\}$

$=1.828+0.001$

$\Rightarrow \mathrm{x}_{3}=1.829$

\section{IV iteration:}

\begin{tabular}{|c|c|c|c|c|}
\hline \multirow[t]{2}{*}{1.829} & 1 & 0 & 24 & -50 \\
\hline & - & 1.829 & 3.345 & 50.014 \\
\hline \multirow[t]{2}{*}{1.829} & 1 & 1.829 & 27.345 & \multirow[t]{5}{*}{0.014} \\
\hline & - & 1.829 & 6.690 & \\
\hline \multirow[t]{3}{*}{1.829} & 1 & 3.658 & \multirow[t]{3}{*}{34.035} & \\
\hline & - & 1.829 & & \\
\hline & 1 & 5.487 & & \\
\hline
\end{tabular}

$\mathrm{f}(1.829)=0.014, \mathrm{f}^{\prime}(1.829)=34.035, \frac{1}{2 !} \mathrm{f}^{\prime \prime}(1.829)=$ $5.487 \Rightarrow \mathrm{f}^{\prime \prime}(1.829)=10.974$

From Modified Newton Raphson method,

$x_{4}=x_{3}-\left\{\frac{f\left(x_{3}\right) f^{\prime}\left(x_{3}\right)}{\left\{f^{\prime}\left(x_{3}\right)\right\}^{2}-f\left(x_{3}\right) f^{\prime \prime}\left(x_{3}\right)}\right\}=1-$

$\left\{\frac{(0.014)(34.035)}{\{34.035\}^{2}-(0.014)(10.974)}\right\}$

$=1.829+0.000$

$\Rightarrow \mathrm{x}_{4}=1.829$

Published By:

Blue Eyes Intelligence Engineering and Sciences Publication (BEIESP) 8 (C) Copyright: All rights reserved. 


\section{Comparative Study of Various Iterative Numerical Methods for Computation of Approximate Root of the Polynomials}

Birge-Vieta Method:

Here $\mathrm{f}(\mathrm{x})=\mathrm{x}^{3}+24 \mathrm{x}-50=0 \Rightarrow \mathrm{f}^{\prime}(\mathrm{x})=3 \mathrm{x}^{2}+24$

We know that $\mathrm{f}(\mathrm{x})=0$ having one of the root in the interval $(1,2)$.

\section{I iteration:}

From the I iteration of Horner's method,

$\mathrm{f}(1)=-25, \mathrm{f}^{\prime}(1)=27$

From Newton Raphson method,

$x_{1}=x_{0}-\left\{\frac{f\left(x_{0}\right)}{f^{\prime}\left(x_{0}\right)}\right\}=1-\left\{\frac{-25}{27}\right\}=1+0.926 \Rightarrow x_{1}=1.926$

\section{II iteration:}

\begin{tabular}{|c|c|c|c|c|}
\hline \multirow{3}{*}{1.926} & 1 & 0 & 24 & -50 \\
\cline { 2 - 4 } & - & 1.926 & 3.709 & 53.368 \\
\hline \multirow{2}{*}{1.926} & 1 & 1.926 & 27.709 & \multirow{2}{*}{3.368} \\
\cline { 2 - 4 } & - & 1.926 & 7.419 & \\
\hline & 1 & 3.852 & 35.128 & \\
\hline
\end{tabular}

$\Rightarrow \mathrm{f}(1.926)=3.368, \mathrm{f}^{\prime}(1.926)=35.128$

From Newton Raphson method,

$$
\begin{aligned}
& \mathrm{x}_{2}=\mathrm{x}_{1}-\left\{\frac{\mathrm{f}\left(\mathrm{x}_{1}\right)}{\mathrm{f}^{\prime}\left(\mathrm{x}_{1}\right)}\right\}=1.926-\left\{\frac{3.368}{35.128}\right\}=1.926-0.096 \\
& \Rightarrow \mathrm{x}_{2}=1.83
\end{aligned}
$$

\section{III iteration:}

\begin{tabular}{|c|c|c|c|c|}
\hline \multirow{2}{*}{1.83} & 1 & 0 & 24 & -50 \\
\cline { 2 - 4 } & - & 1.83 & 3.349 & 50.213 \\
\hline \multirow{2}{*}{1.83} & 1 & 1.83 & 27.349 & 0.213 \\
\cline { 2 - 4 } & - & 1.83 & 6.698 & \\
\hline & 1 & 3.66 & 34.137 & \\
\hline
\end{tabular}

$\Rightarrow \mathrm{f}(1.83)=0.213, \mathrm{f}^{\prime}(1.83)=34.137$

From Newton Raphson method,

$$
\begin{aligned}
& x_{3}=x_{2}-\left\{\frac{f\left(x_{2}\right)}{f^{\prime}\left(x_{2}\right)}\right\}=1.83-\left\{\frac{0.213}{34.137}\right\}=1.83-0.006 \\
& \Rightarrow x_{3}=1.824
\end{aligned}
$$

\section{IV iteration:}

\begin{tabular}{|c|c|c|c|c|}
\hline \multirow{2}{*}{1.824} & 1 & 0 & 24 & -50 \\
\cline { 2 - 4 } & - & 1.824 & 3.327 & 49.844 \\
\hline \multirow{2}{*}{1.824} & 1 & 1.824 & 27.327 & \multirow{2}{*}{-0.156} \\
\cline { 2 - 4 } & - & 1.824 & 6.654 & \\
\hline & 1 & 3.648 & 33.981 & \\
\hline
\end{tabular}

$\Rightarrow \mathrm{f}(1.824)=-0.156, \mathrm{f}^{\prime}(1.824)=33.981$

From Newton Raphson method,

$\mathrm{x}_{4}=\mathrm{x}_{3}-\left\{\frac{\mathrm{f}\left(\mathrm{x}_{3}\right)}{\mathrm{f}^{\prime}\left(\mathrm{x}_{2}\right)}\right\}=1.824-\left\{\frac{-0.156}{33.981}\right\}=1.824+0.005$

$\Rightarrow \mathrm{x}_{4}=1.829$

\section{V iteration:}

\begin{tabular}{|c|c|c|c|c|}
\hline \multirow{2}{*}{1.829} & 1 & 0 & 24 & -50 \\
\cline { 2 - 4 } & - & 1.829 & 3.345 & 50.014 \\
\hline \multirow{2}{*}{1.829} & 1 & 1.829 & 27.345 & \multirow{2}{*}{0.014} \\
\cline { 2 - 4 } & - & 1.829 & 6.690 & \\
\hline & 1 & 3.658 & 34.035 & \\
\hline
\end{tabular}

$\Rightarrow \mathrm{f}(1.829)=0.014, \mathrm{f}^{\prime}(1.829)=34.035$

From Newton Raphson method,

$$
\begin{aligned}
& x_{5}=x_{4}-\left\{\frac{f\left(x_{4}\right)}{f^{\prime}\left(x_{4}\right)}\right\}=1.83-\left\{\frac{0.014}{34.035}\right\}=1.829-0.000 \\
& \Rightarrow x_{5}=1.829
\end{aligned}
$$

Case Study 2: Compute the root of the equation $\mathrm{x}^{3}+x^{2}+3 \mathrm{x}+4=0$.

\section{Solution:}

Horner's method:

Here

$\mathrm{f}(\mathrm{x})=\mathrm{x}^{3}+x^{2}+3 \mathrm{x}+4=0$

Then, $\mathrm{f}(-2)=-6<0$ and $\mathrm{f}(-1)=1>0$

$\Rightarrow \mathrm{f}(\mathrm{x})=0$ having one of the root in the interval $(-2,-1)$. So, change $x$ to $-x$ in $\mathrm{f}(\mathrm{x})$.

we get $-\mathrm{x}^{3}+x^{2}-3 \mathrm{x}+4=0 \Rightarrow \mathrm{x}^{3}-x^{2}+3 \mathrm{x}-4=0$

Let $\mathrm{g}(\mathrm{x})=\mathrm{x}^{3}-x^{2}+3 \mathrm{x}-4=0$

Now negative root of (1) is equal to the positive root of (2) with the sign changedg( $\mathrm{x})=\mathrm{x}^{3}-x^{2}+3 \mathrm{x}-4=0$.

Then, $\mathrm{g}(1)=-1<0$ and $\mathrm{g}(2)=6>0$

$\Rightarrow \mathrm{g}(\mathrm{x})=0$ having one of the root in the interval $(1,2)$.

Hence, the integral (integer) part of the root of $\mathrm{g}(\mathrm{x})=0$ is 1 .

I iteration: Now, using 1 we have to diminish the root of the equation $\mathrm{g}(\mathrm{x})=0$.

\begin{tabular}{|l|c|c|c|c|}
\hline \multirow{2}{*}{1} & 1 & -1 & 3 & -4 \\
\cline { 2 - 4 } & - & -1 & 0 & -3 \\
\hline \multirow{2}{*}{1} & 1 & 0 & 3 & -1 \\
\cline { 2 - 4 } & - & 1 & 1 & \\
& 1 & 1 & 4 & \\
\cline { 2 - 3 } & - & 1 & & \\
\hline & 1 & 2 & & \\
\hline
\end{tabular}

$\mathrm{g}_{1}(\mathrm{x})=\mathrm{x}^{3}+2 \mathrm{x}^{2}+4 \mathrm{x}-1=0$

$\Rightarrow \mathrm{g}_{1}(\mathrm{x})=0$ having a root in the interval $(0,1)$.

Now, multiply the root of $g_{1}(x)=0$ by 10 so that the changed equation $\mathrm{g}_{1}(\mathrm{x})=\mathrm{x}^{3}+20 \mathrm{x}^{2}+400 \mathrm{x}-1000=0$. We see that $g_{1}(2)<0$ and $g_{1}(3)>0$

So, the root of $\mathrm{g}_{1}(\mathrm{x})=0$ which occurs between 2 and 3 . Hence the first figure after the decimal place is 2

II iteration: Now, using 2 we have to diminish the root of the equation $g_{1}(\mathrm{x})=0$

\begin{tabular}{|c|c|c|c|c|}
\hline \multirow{2}{*}{2} & 1 & 20 & 400 & -1000 \\
\cline { 2 - 4 } & - & 2 & 44 & 888 \\
\hline \multirow{2}{*}{2} & 1 & 22 & 444 & -112 \\
\cline { 2 - 4 } & - & 2 & 48 & \\
\cline { 2 - 4 } & 1 & 24 & 492 & \\
\hline & - & 2 & & \\
\hline
\end{tabular}

$g_{2}(x)=x^{3}+26 x^{2}+492 x-112=0$

$\Rightarrow \mathrm{g}_{2}(\mathrm{x})=0$ having a root in the interval $(0,1)$. Now multiply the root of $g_{2}(x)=0$ by 10 . then, the resultant equation $\mathrm{g}_{2}(\mathrm{x})=\mathrm{x}^{3}+260 \mathrm{x}^{2}+49200 \mathrm{x}-112000=0$.

Consider the last two terms by omitting the remaining terms of $g_{2}(x)=0$.

We get, $49200 \mathrm{x}-112000=0 \Rightarrow x=\frac{112000}{49200}=2.28$

We can see that $g_{2}(2)<0$ and $g_{2}(3)>0$. So, the root of $\mathrm{g}_{2}(\mathrm{x})=0$ which occurs between 2 and 3

Hence the second figure after the decimal place is 2 .

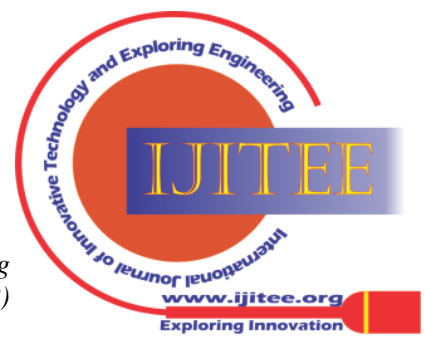


III iteration: Now, using 2 we have to diminish the root of the equation $\mathrm{g}_{2}(\mathrm{x})=0$

\begin{tabular}{|c|c|c|c|c|}
\hline 2 & 1 & 260 & 49200 & -112000 \\
\cline { 2 - 4 } & - & 2 & 524 & 99448 \\
\hline 2 & 1 & 262 & 49724 & -12552 \\
\cline { 2 - 3 } & - & 2 & 528 & \\
\hline 2 & 1 & 264 & 50252 & \\
\cline { 2 - 3 } & - & 2 & & \\
\hline & 1 & 266 & & \\
\hline
\end{tabular}

Thus, the transformed equation $\mathrm{g}_{3}(\mathrm{x})=\mathrm{x}^{3}+266 \mathrm{x}^{2}+50252 \mathrm{x}-12552=0$

$\Rightarrow \mathrm{g}_{3}(\mathrm{x})=0$ having a root in the interval $(0,1)$. Now, multiply the root of $\mathrm{g}_{3}(\mathrm{x})=0$ by 10 then, the equation $\mathrm{g}_{3}(\mathrm{x})=\mathrm{x}^{3}+2660 \mathrm{x}^{2}+5025200 \mathrm{x}-12552000=0$.

Consider the last two terms by omitting the remaining terms of $g_{3}(x)=0$, we get $5025200 x-12552000=0$ $\Rightarrow x=\frac{12552000}{5025200}=2.50$ $\mathrm{g}_{3}(\mathrm{x})=0$ occurs between 2 and 3

Hence the third figure after the decimal place is 2 .

IV iteration: Now, using 2 we have to diminish the root of the equation $\mathrm{g}_{3}(\mathrm{x})=0$

\begin{tabular}{|c|c|c|c|c|}
\hline 2 & 1 & 2660 & 5025200 & -12552000 \\
\cline { 2 - 4 } & - & 2 & 5324 & 10061048 \\
\hline 2 & 1 & 2662 & 5030524 & -2490952 \\
\cline { 2 - 4 } & - & 2 & 5328 & \\
\hline 2 & 1 & 2664 & 5035852 & \\
\cline { 2 - 3 } & - & 2 & & \\
\hline & 1 & 2666 & & \\
\hline
\end{tabular}

Thus, the transformed equation $\mathrm{g}_{4}(\mathrm{x})=\mathrm{x}^{3}+2666 \mathrm{x}^{2}+5035852 \mathrm{x}-2490952=0$

$\Rightarrow \mathrm{g}_{4}(\mathrm{x})=0$ having one of the root in the interval $(0,1)$.

Now, multiply the root of $g_{4}(x)=0$ by 10 then, the resultant equation

$\mathrm{g}_{4}(\mathrm{x})=\mathrm{x}^{3}+26660 \mathrm{x}^{2}+503585200 \mathrm{x}-2490952000=0$

Consider the last two terms by omitting the remaining terms of $g_{4}(x)=0$.

We $\quad$ get, $\quad 503585200 \mathrm{x}-2490952000=0$
$\Rightarrow x=\frac{2490952000}{503585200}=4.95$ We can see that $g_{4}(4)<0$ and $g_{4}(5)>0$. The root of $\mathrm{g}_{4}(\mathrm{x})=0$ which occurs in the interval $(4,5)$

So, the fourth figure after the decimal place is 4 .

Hence, the root of $\mathrm{g}(\mathrm{x})=0$ in the three places of decimals is $1.2224 \cong 1.222$.

Therefore, the root of the given equation $\mathrm{f}(\mathrm{x})=0$ is -1.222

\section{Synthetic division with Chebyshev method:}

Here, $\mathrm{f}(\mathrm{x})=\mathrm{x}^{3}+x^{2}+3 \mathrm{x}+4=0$

Then, $\mathrm{f}(-2)=-6<0$ and $\mathrm{f}(-1)=1>0$

$\Rightarrow \mathrm{f}(\mathrm{x})=0$ having a negative root in the interval $(-2,-2)$. So, change $x$ to $-x$ in $\mathrm{f}(\mathrm{x})$

we get $-\mathrm{x}^{3}+x^{2}-3 \mathrm{x}+4=0 \Rightarrow \mathrm{x}^{3}-x^{2}+3 \mathrm{x}-4=0$

Let $\mathrm{g}(\mathrm{x})=\mathrm{x}^{3}-x^{2}+3 \mathrm{x}-4=0$

Now negative root of (3) is equal to the positive root of (4) with the sign changed

I iteration: $\mathrm{g}(\mathrm{x})=\mathrm{x}^{3}-x^{2}+3 \mathrm{x}-4=0$

$\Rightarrow \mathrm{g}^{\prime}(x)=3 x^{2}-2 x+3$ and $\mathrm{g}^{\prime \prime}(x)=6 x-2$
We can see that $g_{3}(2)<0$ and $g_{3}(3)>0 \Rightarrow$ the root of

$\mathrm{g}(1)=-1, \mathrm{~g}^{\prime}(1)=4, \frac{1}{2 !} \mathrm{g}^{\prime \prime}(1)=2 \Rightarrow \mathrm{g}^{\prime \prime}(1)=4$

Chebyshev method, $x_{1}=x_{0}-\left\{\frac{g\left(x_{0}\right)}{g^{\prime}\left(x_{0}\right)}\right\}-\frac{1}{2}\left[\frac{\left\{g\left(x_{0}\right)\right\}^{2} g^{\prime \prime}\left(x_{0}\right)}{\left\{g^{\prime}\left(x_{0}\right)\right\}^{3}}\right]$

$$
\begin{aligned}
& =1-\left\{\frac{-1}{4}\right\}-\frac{1}{2}\left[\frac{\{-1\}^{2}(4)}{\{4\}^{3}}\right] \\
& =1+0.25-0.031 \Rightarrow x_{1}=1.219
\end{aligned}
$$

\section{II iteration:}

\begin{tabular}{|c|c|c|c|c|}
\hline 1.219 & 1 & 1 & 3 & -4 \\
\cline { 2 - 4 } & - & 1.219 & 0.270 & 3.986 \\
\hline \multirow{2}{*}{1.219} & 1 & 0.219 & 3.270 & -0.014 \\
\cline { 2 - 4 } & - & 1.219 & 1.753 & \\
\hline 1.219 & 1 & 1.438 & 5.023 & \multirow{2}{*}{} \\
\cline { 2 - 3 } & - & 1.219 & & \\
\hline & 1 & 2.657 & & \\
\hline
\end{tabular}

$\mathrm{g}(1.219)=-0.014, \mathrm{~g}^{\prime}(1.219)=5.023$, $\frac{1}{2 !} g^{\prime \prime}(1.219)=2.657 \Rightarrow g^{\prime \prime}(1.219)=5.314$

Chebyshev method: $x_{2}=x_{1}-\left\{\frac{g\left(x_{1}\right)}{g^{\prime}\left(x_{1}\right)}\right\}-\frac{1}{2}\left[\frac{\left[g\left(x_{1}\right)\right\}^{2} g^{\prime \prime}\left(x_{1}\right)}{\left\{g\left(x_{1}\right)\right]^{3}}\right]$

$$
=1.219-\left\{\frac{-0.014}{5.023}\right\}-\frac{1}{2}\left[\frac{\{-0.014\}^{2}(5.314)}{\{5.023\}^{3}}\right]
$$$$
=1.219+0.003-0.000
$$

$\Rightarrow \mathrm{x}_{2}=1.222$

\section{Synthetic division with Modified Newton Raphson} method:

Here $\mathrm{f}(\mathrm{x})=\mathrm{x}^{3}+x^{2}+3 \mathrm{x}+4=0$

$\Rightarrow \mathrm{f}^{\prime}(\mathrm{x})=3 \mathrm{x}^{2}+2 \mathrm{x}+3$ and $\mathrm{f}^{\prime \prime}(\mathrm{x})=6 \mathrm{x}+2$

We know that $f(x)=0$ having a root in the interval $(-2,-1)$

\section{I iteration:}

From the I iteration of Horner's method,

$\mathrm{f}(-1)=1, \mathrm{f}^{\prime}(-1)=4, \frac{1}{2 !} \mathrm{f}^{\prime \prime}(-1)=-2 \Rightarrow \mathrm{f}^{\prime \prime}(-1)=-4$

From Modified Newton Raphson method,

$x_{1}=x_{0}-\left\{\frac{f\left(x_{0}\right) f^{\prime}\left(x_{0}\right)}{\left\{f^{\prime}\left(x_{n}\right)\right]^{2}-f\left(x_{n}\right) f^{\prime \prime}\left(x_{n}\right)}\right\}=-1-\left\{\frac{(1)(4)}{\{4]^{2}-(1)(-4)}\right\}$

$=-1-0.2 \Rightarrow x_{1}=-1.2$

\section{II iteration:}

\begin{tabular}{|c|c|c|c|c|}
\hline-1.2 & 1 & 1 & 3 & 4 \\
\cline { 2 - 4 } & - & -1.2 & 0.24 & -3.888 \\
\hline \multirow{2}{*}{-1.2} & 1 & -0.2 & 3.24 & 0.112 \\
\cline { 2 - 4 } & - & -1.2 & 1.68 & \\
\hline \multirow{2}{*}{1.2} & 1 & -1.4 & 4.92 & \\
\cline { 2 - 3 } & - & -1.2 & & \\
\hline & 1 & -2.6 & & \\
\hline
\end{tabular}

$\mathrm{f}(-1.2)=0.112, \mathrm{f}^{\prime}(-1.2)=4.92, \frac{1}{2 !} \mathrm{f}^{\prime \prime}(-1.2)=-2.6 \Rightarrow$ $\mathrm{f}^{\prime \prime}(-1.2)=-5.2$

From Modified Newton Raphson method,

$$
\begin{aligned}
x_{2} & =x_{1}-\left\{\frac{f\left(x_{1}\right) f^{\prime}\left(x_{1}\right)}{\left\{f^{\prime}\left(x_{1}\right)\right\}^{2}-f\left(x_{1}\right) f^{\prime \prime}\left(x_{1}\right)}\right\}=1-\left\{\frac{(0.112)(4.92)}{\{4.92\}^{2}-(0.112)(-5.2)}\right\} \\
& =-1.2-0.022 \\
\Rightarrow & x_{2}=-1.222
\end{aligned}
$$

Published By:

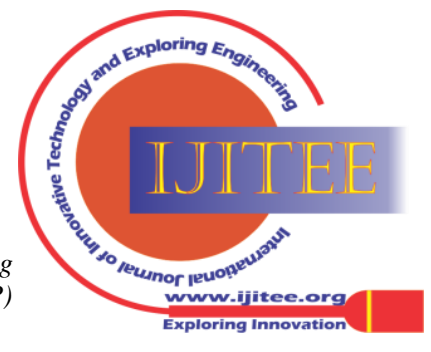


Comparative Study of Various Iterative Numerical Methods for Computation of Approximate Root of the Polynomials

III iteration:

\begin{tabular}{|c|c|c|c|c|}
\hline-1.222 & 1 & 1 & 3 & 4 \\
\cline { 2 - 4 } & - & -1.222 & 0.271 & -3.997 \\
\hline-1.222 & 1 & -0.222 & 3.271 & 0.003 \\
\cline { 2 - 4 } & - & -1.222 & 1.765 & \\
\hline-1.222 & 1 & -1.444 & 5.036 & \\
& - & -1.222 & & \\
\hline & 1 & -2.666 & & \\
\hline
\end{tabular}

$\mathrm{f}(-1.222)=0.003, \mathrm{f}^{\prime}(-1.222)=5.036$,

$\frac{1}{2 !} \mathrm{f}^{\prime \prime}(-1.222)=-2.666 \Rightarrow \mathrm{f}^{\prime \prime}(-1.222)=-5.332$

From Modified Newton Raphson method,

$x_{3}=x_{2}-\left\{\frac{f\left(x_{2}\right) f^{\prime}\left(x_{2}\right)}{\left[f^{\prime}\left(x_{2}\right)\right]^{2}-f\left(x_{2}\right) f^{\prime \prime}\left(x_{2}\right)}\right\}=1-$

$\left\{\frac{(0.003)(5.036)}{\{5.036]^{2}-(0.003)(-5.332)}\right\}$

$=-1.222-0.001$

$\Rightarrow \mathrm{x}_{3}=-1.223$

IV iteration:

\begin{tabular}{|c|c|c|c|c|}
\hline-1.223 & 1 & 1 & 3 & 4 \\
\cline { 2 - 4 } & - & -1.223 & 0.273 & -4.003 \\
\hline \multirow{2}{*}{-1.223} & 1 & -0.223 & 3.273 & -0.003 \\
\cline { 2 - 4 } & - & -1.223 & 1.768 & \\
\hline-1.223 & 1 & -1.446 & 5.041 & \multirow{2}{*}{} \\
\cline { 2 - 3 } & - & -1.223 & & \\
\hline & 1 & -2.669 & & \\
\hline
\end{tabular}

$\mathrm{f}(-1.223)=-0.003, \mathrm{f}^{\prime}(-1.223)=5.041$,

$\frac{1}{2 !} \mathrm{f}^{\prime \prime}(-1.223)=-2.669 \Rightarrow \mathrm{f}^{\prime \prime}(-1.223)=-5.338$

From Modified Newton Raphson method,

$x_{4}=x_{3}-\left\{\frac{f\left(x_{3}\right) f^{\prime}\left(x_{3}\right)}{\left\{f^{\prime}\left(x_{3}\right)\right\}^{2}-f\left(x_{3}\right) f^{\prime \prime}\left(x_{3}\right)}\right\}=1-$

$\left\{\frac{(-0.003)(5.041)}{\{5.041\}^{2}-(-0.003)(-5.338)}\right\}$

$=-1.223-0.001$

$\Rightarrow \mathrm{x}_{4}=-1.222$

\section{Birge-Vieta Method:}

Here $\mathrm{f}(\mathrm{x})=\mathrm{x}^{3}+x^{2}+3 \mathrm{x}+4=0 \Rightarrow \mathrm{f}^{\prime}(\mathrm{x})=3 \mathrm{x}^{2}+2 \mathrm{x}+3$ We know that $f(x)=0$ having one of the root in the interval $(-2,-1)$.

I iteration: From the I iteration of Horner's method,

$\mathrm{f}(-1)=1, \mathrm{f}^{\prime}(-1)=4$

$\mathrm{x}_{1}=\mathrm{x}_{0}-\left\{\frac{\mathrm{f}\left(\mathrm{x}_{0}\right)}{\mathrm{f}^{\prime}\left(\mathrm{x}_{\mathrm{n}}\right)}\right\}=-1-\left\{\frac{1}{4}\right\}=-1.25 \Rightarrow \mathrm{x}_{1}=-1.25$

II iteration:

\begin{tabular}{|c|c|c|c|c|}
\hline-1.25 & 1 & 1 & 3 & 4 \\
\cline { 2 - 4 } & - & -1.25 & 0.313 & -4.141 \\
\hline \multirow{2}{*}{-1.25} & 1 & -0.25 & 3.313 & -0.141 \\
\cline { 2 - 4 } & - & -1.25 & 1.875 & \\
\hline & 1 & -1.50 & 5.188 & \\
\hline
\end{tabular}

$\Rightarrow \mathrm{f}(-1.25)=-0.141, \mathrm{f}^{\prime}(-1.25)=5.188$

From Newton Raphson method,

$\mathrm{x}_{2}=\mathrm{x}_{1}-\left\{\frac{\mathrm{f}\left(\mathrm{x}_{1}\right)}{\mathrm{f}^{\prime}\left(\mathrm{x}_{1}\right)}\right\}=-1.25-\left\{\frac{-0.141}{5.188}\right\}=-1.25+0.027$

$\Rightarrow \mathrm{x}_{2}=-1.223$
From Newton Raphson method:

III iteration:

\begin{tabular}{|c|c|c|c|c|}
\hline III iteration: \\
\hline-1.223 & 1 & 1 & 3 & 4 \\
\cline { 2 - 4 } & - & -1.223 & 0.273 & -4.003 \\
\hline \multirow{2}{*}{-1.223} & 1 & -0.223 & 3.273 & -0.003 \\
\cline { 2 - 4 } & - & -1.223 & 1.768 & \\
\hline & 1 & -1.446 & 5.041 & \\
\hline
\end{tabular}

$\Rightarrow \mathrm{f}(-1.223)=-0.003, \mathrm{f}^{\prime}(-1.223)=5.041$

From Newton Raphson method,

$$
\begin{aligned}
& x_{3}=x_{2}-\left\{\frac{f\left(x_{2}\right)}{f^{\prime}\left(x_{2}\right)}\right\}=-1.223-\left\{\frac{-0.003}{5.041}\right\}=-1.223+0.001 \\
& \Rightarrow x_{3}=-1.223
\end{aligned}
$$

\section{COMPARISON TABLE}

Table I: Number of iterations performed to compute the approximate root of the polynomial equations in the above

\begin{tabular}{|c|c|c|c|c|}
\hline \multicolumn{5}{|c|}{ Number of iterations } \\
\hline 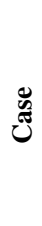 & 葛 & 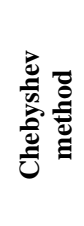 & 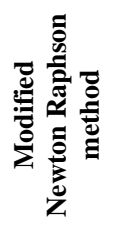 & 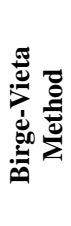 \\
\hline 1 & 4 & 3 & 4 & 5 \\
\hline 2 & 4 & 2 & 4 & 3 \\
\hline
\end{tabular}
case study

\section{CONCLUSION}

While computing the approximate root of the polynomial equations using many methods in the above case study, it is observed that Synthetic Division with Chebyshev Method (Proposed Method) is converged quickly compared to other three methods. Also, it requires less time consumption in performing the iterations to compute approximate root of the polynomial equations.

\section{REFERENCES}

1. Comparative analysis of polynomial root finding techniques; STUDENT THESES AND DISSERTATIONS, 1967: Charles Ray O'Daniel

2. Mathematical Model Formulation and Comparison Study of Various Methods of Root- Finding Problems; IOSR Journal of Mathematics (IOSR-JM); Arup Dey; e-ISSN: 2278-5728, p-ISSN: 2319-765X. Volume 11, Issue 2 Ver. III (Mar - Apr. 2015), PP 64-71 www.iosrjournals.org

3. Comparative Study of Bisection, Newton-Raphson and Secant Methods of Root- Finding Problems; Ehiwario, J.C., Aghamie, S.O.; IOSR Journal of Engineering (IOSRJEN) ISSN (e): 2250-3021, ISSN (p): 2278-8719

4. Text Book-Numerical methods by R. K Jain and S.R.K Iyengar

\section{AUTHORS PROFILE}

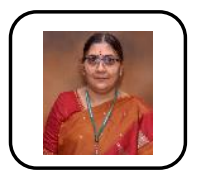

Dr. Roopa KM, M.Sc., M.Phil., Ph.D. Professor, Department of Mathematics, Dean for Skil Development, Bangalore Institute of Technology, Bengaluru, Published 10 Papers in National and International Journals.

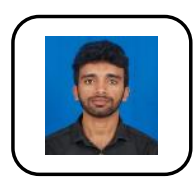

Venkatesha P, M.Sc., (Ph.D). Assistant Professor, Department of Mathematics, Sri Sairam College of Engineering, Bengaluru.

Published By:

Blue Eyes Intelligence Engineering and Sciences Publication (BEIESP) 